Article

\title{
Effectiveness of a Real-Time Ventilation Feedback Device for Guiding Adequate Minute Ventilation: A Manikin Simulation Study
}

\author{
Sejin Heo ${ }^{1}$, Sun Young Yoon ${ }^{1}$, Jongchul Kim ${ }^{2}$, Hye Seung Kim ${ }^{3}$, Kyunga Kim ${ }^{3,4}$, Hee Yoon ${ }^{1}$, \\ Sung Yeon Hwang ${ }^{1}{ }^{\mathbb{D}}$, Won Chul Cha ${ }^{1,4}$ and Taerim Kim ${ }^{1, *}$ \\ 1 Department of Emergency Medicine, Samsung Medical Center, Sungkyunkwan University School of \\ Medicine, Seoul 06351, Korea; silversh06@naver.com (S.H.); roseherb21@naver.com (S.Y.Y.); \\ wildhi.yoon@samsung.com (H.Y.); sygood.hwang@samsung.com (S.Y.H.); wc.cha@samsung.com (W.C.C.) \\ 2 Department of Biomedical Engineering, Samsung Medical Center, Seoul 06351, Korea; \\ goodman0707.kim@samsung.com \\ 3 Biostatistics and Clinical Epidemiology Center, Samsung Medical Center, Seoul 06351, Korea; \\ hyeseung.kim@sbri.co.kr (H.S.K.); Kyunga.j.kim@samsung.com (K.K.) \\ 4 Department of Digital Health, Samsung Advanced Institute for Health Sciences \& Technology, \\ Sungkyunkwan University, Seoul 06355, Korea \\ * Correspondence: taerim.j.kim@gmail.com; Tel.: +82-2-3410-2053; Fax: +82-2-3410-0049
}

Received: 17 April 2020; Accepted: 1 June 2020; Published: 5 June 2020

check for updates

\begin{abstract}
Background and objectives: It is often challenging even for skilled rescuers to provide adequate positive pressure ventilation consistently. This study aimed to investigate the effectiveness of a newly developed real-time ventilation feedback device (RTVFD) that estimates tidal volume (TV) and ventilation interval (VI) in real time. Materials and methods: We conducted a randomised, crossover, manikin simulation study. A total of 26 medical providers were randomly assigned to the RTVFD-assisted ventilation (RAV) first group $(n=13)$ and the non-assisted ventilation (NV) first group $(n=13)$. Participants provided ventilation using adult and paediatric bag valves (BVs) for 2 min each. After a washout period, the simulation was repeated by exchanging the participants' groups. Results: The primary outcome was optimal TV in the RAV and NV groups using adult and paediatric BVs. A secondary outcome was optimal VI in the RAV and NV groups using adult and paediatric BVs. The proportions of optimal TV values were higher for the RAVs when using both adult and paediatric BVs (adult BV: $47.29 \%$ vs. $18.46 \%, p<0.001$; paediatric BV: $89.51 \%$ vs. $72.66 \%, p<0.001)$ than for the NVs. The proportions of optimal VI were significantly higher in RAVs when using both adult and paediatric BVs than that in NVs (adult BV: $95.64 \%$ vs. $50.20 \%$, $p<0.001$; paediatric BV: $95.83 \%$ vs. $57.14 \%, p<0.001$ ). Additionally, we found that with paediatric BVs, the simulation had a higher OR for both optimal TV $(13.26 ; 95 \%$ CI, 9.96-17.65; $p<0.001)$ and VI $(1.32 ; 1.08-1.62, p=0.007)$, regardless of RTVFD use. Conclusion: Real-time feedback using RTVFD significantly improves the TV and VI in both adult and paediatric BVs in a manikin simulation study.
\end{abstract}

Keywords: positive pressure ventilation; bag valve; cardiopulmonary resuscitation; simulation study

\section{Introduction}

Despite the continuous efforts to improve the survival rate of cardiac arrest patients, the rate remains poor worldwide [1-4]. Prompt delivery of high-quality cardiopulmonary resuscitation (CPR) ensures a significant survival benefit, according to previous studies $[5,6]$. The American Heart Association (AHA) and European Resuscitation Council guidelines recommend the performance of chest compression at an adequate rate and depth, with minimal pauses between compressions and 
with adequate ventilation, namely, optimal tidal volume (TV) and ventilation interval (VI) to produce a visible chest rise [7-10].

The risks of hyperventilation during CPR, such as decreased cardiac output with increased intrathoracic pressure and decreased cerebral blood due to reduced partial pressure of carbon dioxide in the blood, are well known [11-14]. Thus, the AHA guidelines for CPR recommend an optimal ventilation rate of $10 / \mathrm{min}$ and TV of $6-7 \mathrm{~mL} / \mathrm{kg}$ in both adult and paediatric patients $[10,15]$. However, it is often challenging for rescuers to consistently provide adequate positive ventilation using bag valves (BVs) [16-18]. Hyperventilation, even with professional rescuers, has been reported $[1,13,14,19]$. The simple manipulating method of BV does not guarantee consistently adequate TV [20].

To avoid suboptimal ventilation, some ancillary devices have been developed and studied; the use of a metronome improved the proportions of correct chest compression and ventilation rates, but it could not monitor TV during CPR [21]. A few studies have shown that paediatric BVs could maintain an acceptable level of oxygenation and suggested that adult BVs should be replaced with paediatric BVs during training to prevent hyperventilation [22,23]. The use of an impedance threshold device improved the haemodynamics and short-term survival but could not provide information about the actual TV and failed to prolong long-term survival $[14,24,25]$. Other equipment has been manufactured in recent years $[26,27]$. One of instrument could simultaneously display TV and a bagging time alarm, but TV was only shown through a bar graph variation and was not displayed as the actual numerical volume [26].

To overcome the limitations of the previous devices, we developed a new real-time ventilation feedback device (RTVFD). It displays numerical TV and a bagging time alarm in real time, thus guiding rescuers to achieve optimal ventilation per minute. This study primarily aimed to investigate whether RTVFD increases the proportion of adequate TV when using BVs of different sizes: adult and paediatric BVs. In addition, we compared the proportion of adequate VI with or without the assistance of RTVFD using adult and paediatric BVs.

\section{Methods}

\subsection{A Real-Time Ventilation Feedback Device}

The experimental device was composed of the main board (control board), flow sensor, pressure sensor, organic light-emitting diode display, Bluetooth module, and power (Figure 1A). A controller based on the Arduino Uno Board was created and modified by partial boards for miniaturisation.

A processor sampled three analogue signals at a sampling frequency of $40 \mathrm{~Hz}(25 \mathrm{~ms})$ using the Microchip's ATmega328/P in the process of quantisation and converted analogue to digital at 10 bits. The analogue input value of the processor consisted of a mass flow sensor's (PMF4103V, POSIFA Microsystems Inc., San Jose, CA, USA output value, a pressure sensor's (XGZP6847010KPG, CFSensor Inc., Anhui, China) output value, and the internal battery's (TW632570, 3.7 V, $1200 \mathrm{mAh}$, The HAN Inc., Seoul, Korea) voltage value.

The processor repeated the measurement of the mass flow sensor's output value. The air content of the mass flow sensor was measured 70 times (every $25 \mathrm{~ms}$ ), and the accumulated values were used to obtain an input value of $1.75 \mathrm{~s}$ in the controller. Subsequently, the amount of air was computed by utilising the output formula provided by the manufacturer:

$$
\text { PMF4103V flow rate }=((\text { Vout }-1 \mathrm{~V}) / 4 \mathrm{v}) \times \text { full-scale flow rate }
$$

When the mass flow sensor detected more than a fixed quantity of air, the processor measured the air content and activated two internal timers. The timer measured the time until the next influx to compute the interval of BV ventilation (interval time). The other timer informed the user of the bagging time in real time using the numeric value and an alarm sound (Figure 1B). Moreover, Bluetooth was embedded to link the device with smart devices that measured bagging pressure and air volume in real time through an exclusive application, and this was provided to the users. 


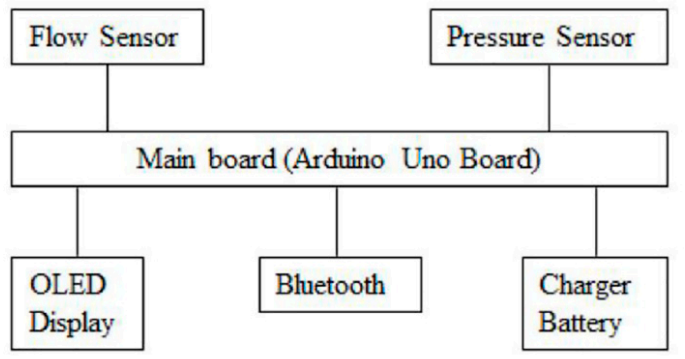

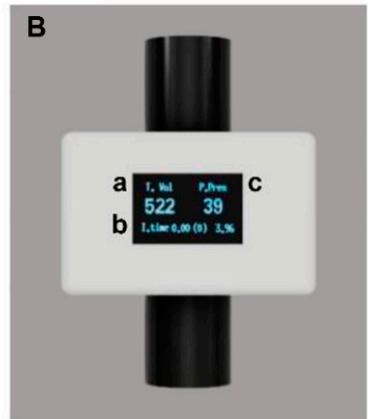

D
C

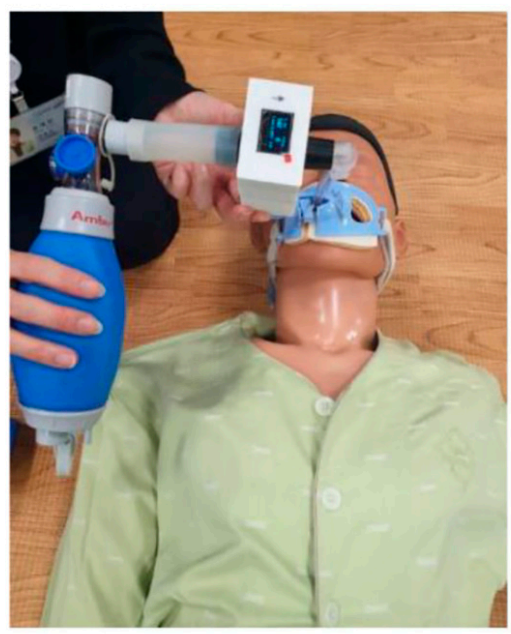

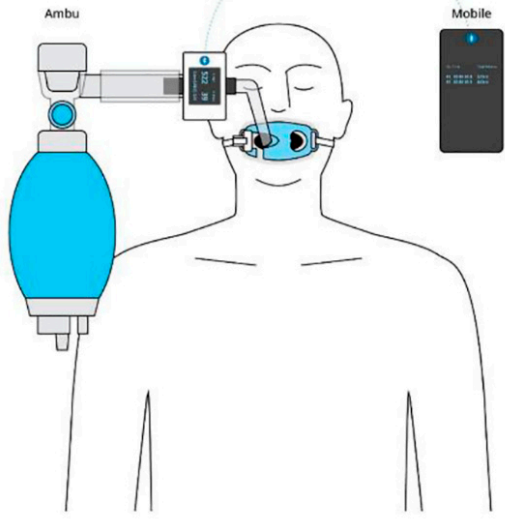

Figure 1. A real-time ventilation feedback device: (A) system overview; (B) real-time ventilation feedback device (RTVFD)—a: tidal volume, b: inspiration time, counted time right before bagging (seconds to next bagging), residual battery, c: peak pressure; $(\mathrm{C})$ paediatric bag valve mask used for manikin simulation; (D) schematic diagram of RTVFD setting. RTVFD, real-time ventilation feedback device.

\subsection{Device Validation}

The RTVFD was connected to a mechanical ventilator (Hamilton G5) with a volume-controlled mode (inspiration/expiration ratio of 1:5 and a respiration rate of 10/min) at TVs of 150, 250, 350, 450, and $550 \mathrm{~mL}$. We provided each TV to the RTVFD using an analyser (gas flow analyser, FLUKE Corps, Washington, USA) 25 times. Subsequently, we measured the mean, standard deviation (SD), and the difference in the ratios of TVs between the RTVFD and the analyser.

$$
\text { Difference in the ratio of TVs }=\frac{T V \text { of } R T V F D-T V \text { of analyser }}{T V \text { of analyser }}
$$

\subsection{Study Design}

We conducted a randomised, crossover, manikin simulation study for a study period from 1 January 2019 to 1 February 2019 at the emergency department of Samsung Medical Center, a tertiary teaching hospital with 250 emergency visits per day. This study was approved by the institutional review board (IRB) of our institute (IRB number: 2018-05-144) on 14 June 2018.

\subsection{Participants}

Based on the pilot study with and without RTVFD using adult BVs, we calculated a sample size of 26 participants per group using the McNemar test for two paired proportions with 2.5\% significance 
and $80 \%$ power. The proportion of discordant pairs was $50 \%$; based on that, the proportion of optimal TV of the RTVFD-assisted ventilation (RAV) was 35\%, and that of the non-assisted ventilation (NV) was $85 \%$. We hypothesised that there is no difference in the proportion of optimal TV provided by adult and paediatric BVs. Participants were recruited by means of recruitment information posted on the employee bulletin board or hospital intranet of the study site. All the participants were medical providers such as doctors, nurses, and emergency medical technicians (EMTs) who were Advanced Cardiovascular Life Support (ACLS)-certified and at the time working in emergency or critical care units. Individuals who have a hearing disorder or musculoskeletal disease were excluded.

\subsection{Study Protocol}

All participants were provided 30 minutes of introduction to the study protocol and the optimal ventilation recommended by the AHA guidelines for CPR. In the first trial, a total of 26 volunteers were randomly assigned to the RAV first group $(n=13)$ and the NV first group $(n=13)$. We used the block randomisation method. The RAV first group was asked to perform ventilation as per the RTVFD guideline, and the NV first group followed the AHA guideline.

The manikin (Ambu ${ }^{\circledR}$ Man Advanced-Next Generation) was intubated using a cuffed endotracheal tube (ETT) with a $7.5 \mathrm{~mm}$ inner diameter, and the depth of ETT placement was $21 \mathrm{~cm}$. The RTVFD was connected between the BV and endotracheal tube (Figure 1C). We applied two different sizes of BVs (Ambu ${ }^{\circledR}$ Mark IV (reusable resuscitator): Ambu Mark IV Adult and Ambu Mark IV Baby). Participants provided ventilation using adult and paediatric BVs for 2 minutes each, with a 2-min break time in between. We measured TV and VI for a total of 4 minutes. The display of the RTVFD was covered while performing non-feedback-assisted ventilation and uncovered during RTVFD feedback-assisted ventilation. TV and VI data were transferred to the application in a handheld device via the Bluetooth of the RTVFD (Figure 1D). We hypothesised that the advanced airway had been secured. This study focused on ventilations only; therefore, chest compressions were not performed.

The optimal TV was defined as $420-480 \mathrm{~mL}$ for adult BVs and $120-180 \mathrm{~mL}$ for paediatric BVs. We set up a similar volume interval $(60 \mathrm{~mL})$ for adult and paediatric BVs. The maximum volumes delivered for an adult BV and paediatric BV were 1300 and $300 \mathrm{~mL}$, respectively [28]. The optimal VI was defined as a breath every 6-8 s. After a 1-week washout period, the simulation was repeated with the participants crossed over to the other group. The flow diagram for the study is shown in Figure 2.

\subsection{Outcomes}

The primary outcome was the proportion of optimal TV in the RAV and NV groups using adult and paediatric BVs. The secondary outcome was the proportion of optimal VI in the RAV and NV groups using adult and paediatric BVs.

\subsection{Statistics}

The baseline characteristics of the two groups were described. Continuous variables with normal distributions were described as means with $\mathrm{SD}$, and those with non-normal distributions were described as medians with interquartile ranges (IQRs). Categorical variables were presented as frequencies with percentages. Student's t-test was used to compare the means of two continuous variables with normal distributions, and the Wilcoxon rank-sum test was used to compare the means of two groups of variables not normally distributed. The frequencies of categorical variables were compared using the chi-square test. We used generalised estimating equation (GEE) models, extensions from logistic regression models, to analyse binary response variables that were repeatedly measured across multiple time points. In the GEE models, the response variables were the achievement status of adequate TV and that of VI. The use of RTVFD assistance and BV type were included as independent variables, and the interaction effect was also examined along with their main effects. Subgroup analyses were further conducted for each BV type. Results for the GEE are reported as odds ratios (ORs) with 95\% confidence intervals (CIs). Differences were considered significant at a 95\% level $(p<0.05)$. Statistical 
analysis was performed using Statistical Analysis System (SAS) version 9.4 (SAS Institute, Cary, NC) and R 3.5.1 (R Foundation for Statistical Computing).

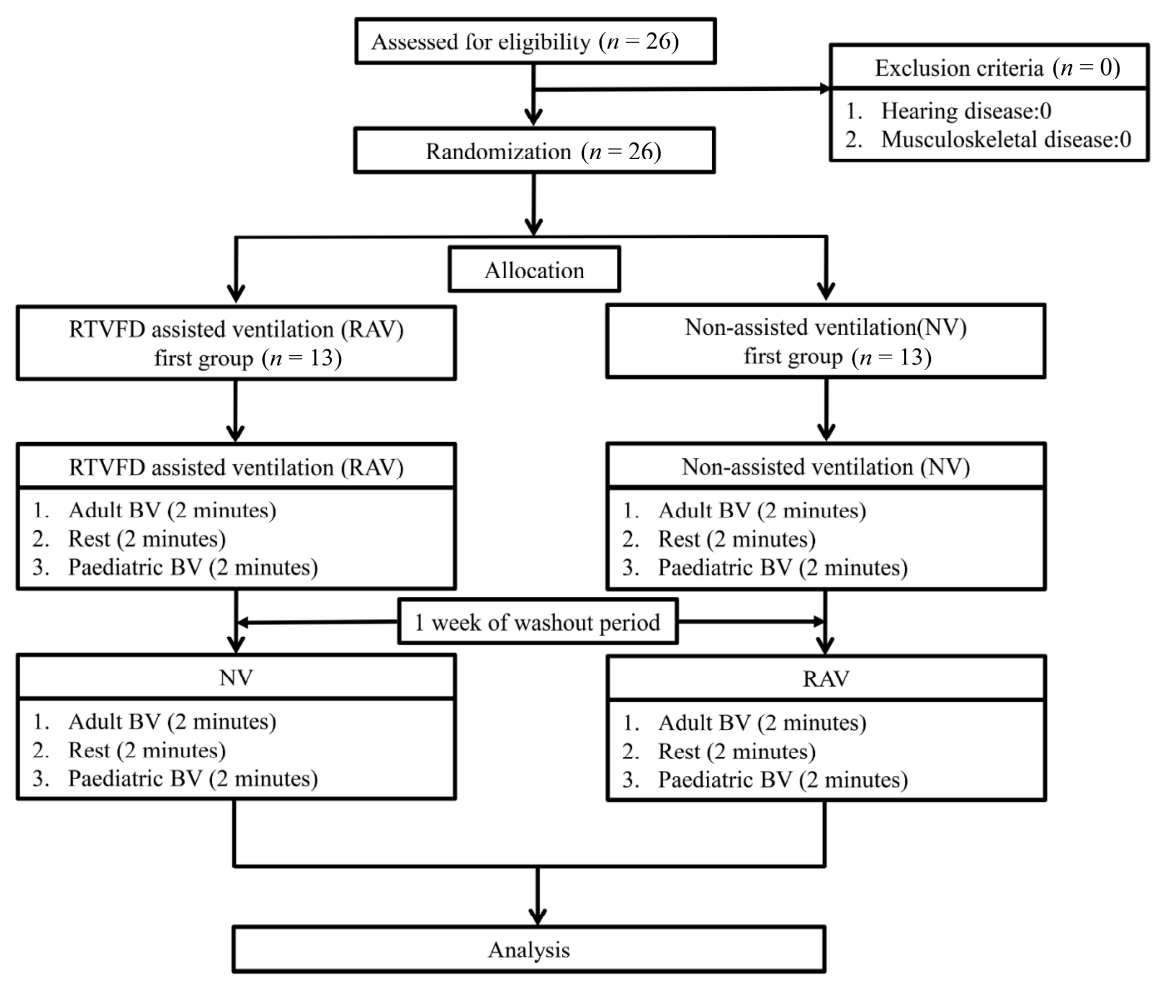

Figure 2. CONSORT flow diagram of the study. RTVFD, real-time ventilation feedback device; BV, bag valve; RAV, real-time ventilation feedback device ventilation; $\mathrm{NV}$, non-assisted ventilation.

\section{Results}

\subsection{Real-Time Ventilation Feedback Device Validation}

The mean \pm SD values of TV in the RTVFD and the analyser are shown in Table 1 and Figure 3. The SD of TVs in the RTVFD was larger than that of the analyser at all target TVs. No SD exceeded 13.7 and $2.2 \mathrm{~mL}$ for the RTVFD and analyser, respectively (Table 1). Among the 125 values of the differences in TVs between the two devices, $109(87.2 \%)$ and 117 (93.6\%) were within $\pm 5 \%$ and $\pm 10 \%$, respectively.

Table 1. Real-time ventilation feedback device validation data.

\begin{tabular}{cccc}
\hline Set Volume of Mechanical Ventilation, $\mathbf{~ m L}$ & Analyser & RTVFD & $p$-Value \\
\hline 150 & $149.4 \pm 1.0$ & $144 \pm 9.8$ & 0.008 \\
250 & $253.9 \pm 0.3$ & $244.3 \pm 9.7$ & $<0.001$ \\
350 & $348.2 \pm 0.6$ & $348.0 \pm 10.9$ & 0.903 \\
450 & $455.8 \pm 1.3$ & $450.1 \pm 9.1$ & 0.005 \\
550 & $551.2 \pm 2.2$ & $545.2 \pm 13.7$ & 0.040 \\
\hline
\end{tabular}

RTVFD, real-time ventilation feedback device. Values are mean \pm SD.

\subsection{Baseline Characteristics}

Twenty-six participants were enrolled and randomly assigned to the two groups: 19 participants were nurses, 4 were EMTs, and 3 were emergency physicians. Table 2 presents the baseline characteristics of the participants. There were no significant differences between the two groups in terms of age, sex, experience, or job. 

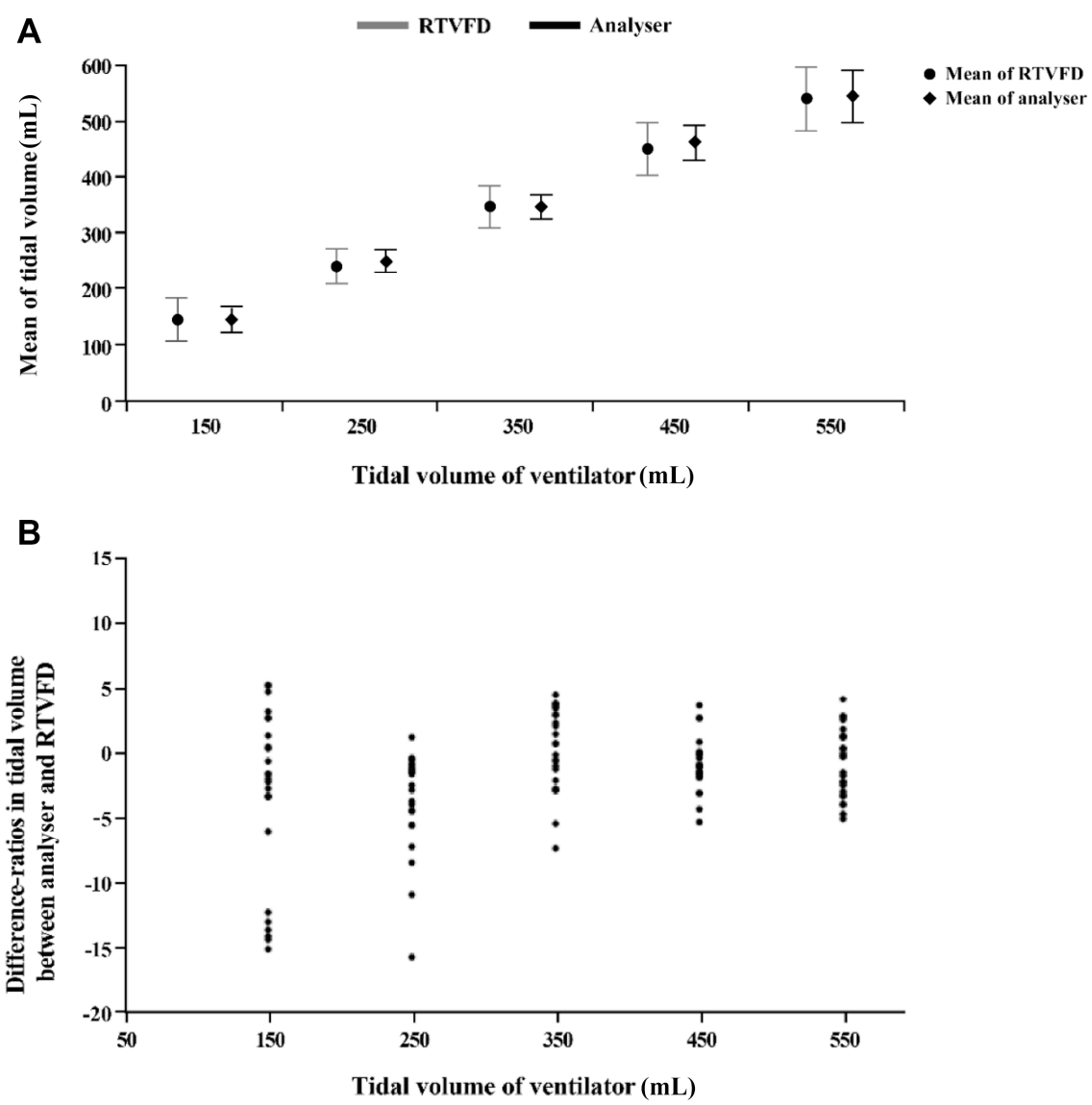

Figure 3. Validation data of RTVFD: (A) mean of tidal volume; (B) difference ratios in tidal volume between analyser and RTVFD. Difference ratios are defined as the tidal volume of RTVFD-tidal volume of analyser/tidal volume of analyser. RTVFD, real-time ventilation feedback device.

Table 2. Baseline characteristics between the two groups.

\begin{tabular}{cccc}
\hline & $\begin{array}{c}\text { RAV First Group } \\
(\boldsymbol{n}=\mathbf{1 3})\end{array}$ & $\begin{array}{c}\text { NV First Group } \\
(\boldsymbol{n}=\mathbf{1 3})\end{array}$ & $\boldsymbol{p}$-Value \\
\hline Age, median (IQR) & $30(27-33)$ & $28(26-30)$ & 0.079 \\
Female, N (\%) & $9(69.2 \%)$ & $8(61.5 \%)$ & 1.000 \\
Times CPR performed & & & \\
Number within 1 week & $0.85(0.80)$ & $1.15(1.46)$ & 0.512 \\
Number within 1 month & $2.15(2.27)$ & $5.31(7.04)$ & 0.137 \\
Job, N (\%) & $10(76.9)$ & $9(69.2)$ & \\
Nurse & $2(15.4)$ & $2(15.4)$ & \\
EMT & $1(7.7)$ & $2(15.4)$ & \\
Doctor & &
\end{tabular}

$\mathrm{RAV}$, real-time ventilation feedback device ventilation; $\mathrm{NV}$, non-assisted ventilation; IQR, interquartile range; $\mathrm{SD}$, standard deviation; EMT, emergency medical technician.

\subsection{Results for the Tidal Volume and Ventilation Interval Performance}

The results for the TV and VI performance are shown in Table 3. The proportions of optimal TV values were significantly higher for the RAVs when using both adult and paediatric BVs (adult BV: $47.29 \%$ vs. $18.46 \%, p<0.001$; paediatric BV: $89.51 \%$ vs. $72.66 \%, p<0.001$ ) (Figure 4 A) than for the NVs. 
Table 3. The results for the tidal volume and ventilation interval performance.

\begin{tabular}{|c|c|c|c|c|c|c|}
\hline & \multicolumn{2}{|c|}{ Adult BV } & \multirow{2}{*}{$p$-Value } & \multicolumn{2}{|c|}{ Paediatric BV } & \multirow{2}{*}{$p$-Value } \\
\hline & RAV & NV & & RAV & NV & \\
\hline Tidal volume & & & $<0.001$ & & & $<0.001$ \\
\hline Tidal volume, $\mathrm{mL}$, mean $\pm \mathrm{SD}$ & $432.0 \pm 63.93$ & $392.83 \pm 136.36$ & & $144.84 \pm 23.07$ & $131.74 \pm 38.78$ & \\
\hline Optimal tidal volume & & & $<0.001$ & & & $<0.001$ \\
\hline Optimal ventilation, N (\%) & $262(47.29)$ & 98 (18.46) & & 495 (89.51) & $380(72.66)$ & \\
\hline Hypoventilation, N (\%) & $220(39.71)$ & $309(58.19)$ & & $53(9.58)$ & $131(25.05)$ & \\
\hline Hyperventilation, N (\%) & $72(13.00)$ & $124(23.35)$ & & $5(0.90)$ & $12(2,29)$ & \\
\hline Ventilation interval & & & $<0.001$ & & & $<0.001$ \\
\hline Ventilation interval, sec & $6.84 \pm 1.13$ & $6.37 \pm 3.17$ & & $6.57 \pm 0.99$ & $6.29 \pm 2.23$ & \\
\hline Optimal interval & & & $<0.001$ & & & $<0.001$ \\
\hline Optimal interval, N (\%) & $505(95.64)$ & $254(50.20)$ & & $506(95.83)$ & $284(57.14)$ & \\
\hline
\end{tabular}

$\mathrm{BV}$, bag valve; RAV, real-time ventilation feedback device ventilation; $\mathrm{NV}$, non-assisted ventilation.

A

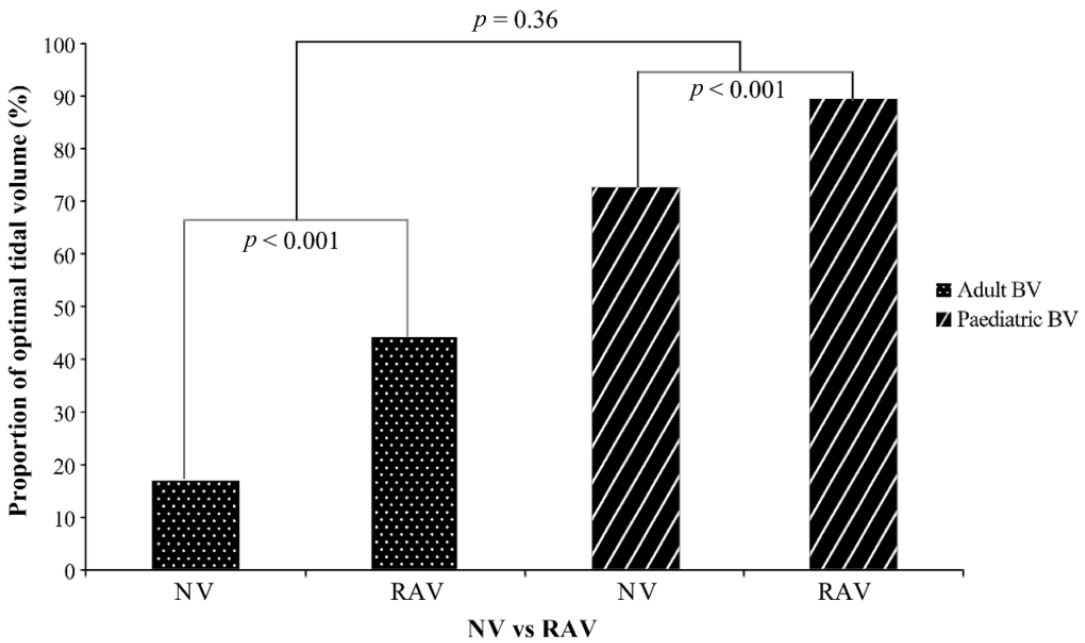

B

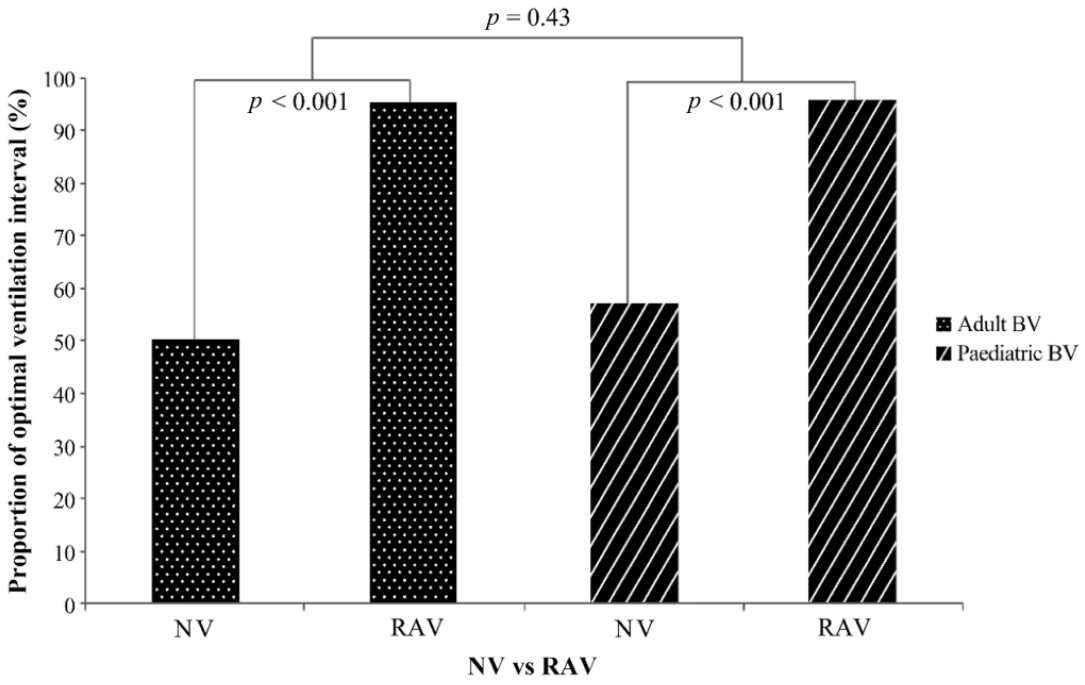

Figure 4. Proportion of optimal TV and VI(\%). (A) Proportion of optimal TV(\%). Comparison of the real-time feedback device-assisted ventilations and the non-assisted ventilations ( $n=554$ for adult, $n=553$ for paediatric BVs) based on BV type. (B) Proportion of optimal VI(\%). Non-assisted ventilations ( $n=506$ for adult, $n=497$ for paediatric BVs) vs. RTVFD-assisted ventilations $(n=528$ for both adult and paediatric BVs) TV, tidal volume; BV, bag valve; RTVFD, real-time ventilation feedback device; $\mathrm{RAV}$, real-time ventilation feedback device ventilation; $\mathrm{NV}$, non-assisted ventilation. 
The mean TV of the RAVs and NVs using adult BVs and paediatric BVs both showed significant differences (adult BV: RAVs, 432.00 (63.93) vs. NVs 392.83 (136.36), $p<0.001$; paediatric BV: RAVs, 144.84 (23.07) vs. NVs 131.74 (38.78), $p<0.001$ ). TV variations were smaller for the RAVs than the NVs.

The proportions of optimal VI were significantly higher in RAVs when using both adult and paediatric BVs than that in NVs (adult BV: 95.64\% vs. 50.20\%, $p<0.001$; paediatric BV: $95.83 \%$ vs. $57.14 \%, p<0.001$ ) (Figure 4B). The mean VI of the RAVs and NVs using adult BV showed a significant differences with both adult and paediatric BVs (adult BV: RAVs, 6.84 (1.13) vs. NVs 6.37 (3.17), $p<0.001$; paediatric BV: RAVs, 6.57 (0.99) vs. NVs 6.29 (2.23), $p<0.001)$. The VI variations of the RAVs were lower than those of NVs.

In the GEE analysis, the OR of optimal TV for the RAVs was 3.90 (95\% confidence interval (CI), 2.95-5.15) regardless of the BV type (Table 4). The ORs of the TV were 3.90 (2.95-5.15) and 3.21 (2.30-4.48) for the adult and paediatric BV groups, respectively. The OR of optimal VI for the RAVs was 21.78 (13.71-34.61) regardless of BV type. The ORs of VI were 21.78 (13.71-34.61) and 17.25 (10.80-27.56) for the adult and paediatric BV groups, respectively.

Table 4. The results from the generalised estimating equation model for evaluating the use of RTVFD and bag valve type.

\begin{tabular}{lcccc}
\hline & Odds Ratio & \multicolumn{2}{c}{$\mathbf{9 5 \%}$ CI } & $p$-Value \\
\hline Achievement of adequate TV & & & & $<0.001$ \\
RTVFD assistance (yes vs. no) & 3.90 & 2.95 & 5.15 & $<0.001$ \\
BV type (paediatric vs. adult BV) & 13.26 & 9.96 & 17.65 & $<0.001$ \\
RTVFD assistance (in adult BV subgroup) & 3.90 & 2.95 & 5.15 & $<0.001$ \\
RTVFD assistance (in paediatric BV subgroup) & 3.21 & 2.30 & 4.48 & 0.364 \\
Interaction (RTVFD assistance \& BV type) & 0.82 & 0.54 & 1.25 & \\
\hline Achievement of adequate VI & & & & $<0.001$ \\
RTVFD assistance (yes vs. no) & 21.78 & 13.71 & 34.61 & 0.007 \\
BV type (paediatric vs. adult BV) & 1.32 & 1.08 & 1.62 & $<0.001$ \\
RTVFD assistance (in adult BV subgroup) & 21.78 & 13.71 & 34.61 & $<0.001$ \\
RTVFD assistance (in paediatric BV subgroup) & 17.25 & 10.80 & 27.56 & 0.430 \\
Interaction (RTVFD assistance \& BV type) & 0.79 & 0.44 & 1.41 & \\
\hline
\end{tabular}

$\mathrm{TV}$, tidal volume; VI, ventilation interval; RTVFD, real-time ventilation feedback device; $\mathrm{BV}$, bag valve; $\mathrm{CI}$, confidence interval; RAV, real-time ventilation feedback device ventilation; $N V$, non-assisted ventilation.

In subgroup analysis, the ORs of ventilations using paediatric BVs were higher (13.26 (9.96-17.65) for TV and 1.32 (1.08-1.62) for VI) than that of adult BVs. We conducted interaction analyses between RTVFD use (with or without) and BV type. The results showed that the effect of RAVs was insignificantly different among the types of BVs with regard to TV and VI.

\section{Discussion}

The present study demonstrated that BV ventilation with RTVFD markedly increased the proportion of optimal TV and VI during manikin simulation. Our device could display the numerical TV repeatedly, even at a small volume for paediatric ventilation, with the alarm for the ventilation cue sounding just in time. This study showed higher ORs for RAVs than for NVs; the OR of optimal VI was higher than that of optimal TV with both adult and paediatric BVs. We set the same optimal volume interval $(60 \mathrm{~mL})$; we found that with paediatric BVs, the simulation had a higher OR for both optimal TV and VI, regardless of RTVFD use.

A BV is generally designed with one-third to half of the bag's entire volume provided at one grasping [16]. However, even trained rescuers experience difficultly in maintaining adequate ventilation, and several studies in this field have emphasised consistent education and training [29,30]. To increase the optimal ventilation, some feedback devices are manufactured, but most of these devices are not applicable to CPR. However, suggestions regarding the educational effect of ancillary devices 
have been reported in previous studies [27,31]. Using feedback devices such as RTVFD and choosing a $\mathrm{BV}$ of adequate size will effectively guide rescuers to deliver optimal ventilation.

Real-time feedback for TV and VI from our device might be useful for professionals to avoid suboptimal ventilation and high respiratory rate. The embedded Bluetooth facilitated the transfer of information to users through an exclusive application. All volunteers could review their own ventilation records after 2 minutes of ventilation. If this device can help professionals to achieve better ventilation performance, non-medical volunteers might benefit as well, though further studies would have to verify that it is still accurate with non-medical staff.

Some studies have suggested the use of a TV feedback device using a turbine flow meter. However, the rotation of the turbine in high TV persists over 6-10 s, which could result in difficulty providing real-time feedback [32]. Another study suggested a flow meter consisting of a round magnet and a spring. However, the difference ratio of TV between the mechanical ventilator and TV device increases during high-TV ventilation because of mechanical friction between the magnet and inner wall of the main body of the TV device. The device we developed is based on the Arduino Uno Board created and modified by partial boards for miniaturisation using a mass flow sensor. This device could measure a wide range of TV values even in a short duration, keeping consistent the difference TV ratio. It could be superior to other equipment in that it displays numerical TV in real time.

Using an RTVFD allows for the appropriate selection of the BV size for adequate TV for patient age and size. A few studies demonstrated that paediatric BVs could maintain adequate oxygenation in adult patients and proposed using paediatric BVs for training to prevent hyperventilation [22,23]. Providing appropriate and consistent volume delivery to adult patients is possible with a smaller, paediatric-sized BV than with an adult BV $[23,33]$. When the TV was below the recommended level, the paediatric self-inflatable bag was able to reduce gastric inflation while ensuring sufficient lung ventilation as compared to the adult self-inflatable bag [34]. This means that choosing a mechanically and structurally appropriate BV is important to achieve optimal ventilation. Our novel device would overcome the structural limitation of the BV and facilitate optimal ventilation.

The present study has some limitations. First, in the device validation study, the SD of RTVFD was higher than that of the analyser for all TVs, specifically the SD of RTVFD, which was greater than $10 \%$ at $550 \mathrm{~mL}$ TV. However, our target was $420-480 \mathrm{~mL}$ TV for adult BV simulation, and regardless of the RTVFD use, the mean TVs were less than $450 \mathrm{~mL}$ with both BV sizes. The difference in the ratios between the analyser and RTVFD was almost within $\pm 10 \%$. However, at the relatively low TV of the ventilator, the difference in ratios was increased. The device could be improved by enhancing precision at both extremes. Second, we did not perform chest compressions on the manikin; therefore, the variation in airway resistance or compliance during chest compression was not reflected. Further studies are needed to verify the accuracy of RTVFD in real-life situations.

\section{Conclusions}

Real-time feedback using RTVFD improved TV and optimal VI with both adult and paediatric BVs in a manikin simulation study. Our novel device could overcome the structural limitations of the BV and facilitate optimal ventilation.

Author Contributions: Conceptualisation, T.K., W.C.C.; methodology, S.H., T.K., W.C.C.; validation, S.H., T.K.; formal analysis, K.K., H.S.K., T.K.; investigation, S.H., S.Y.Y.; resources, S.H., J.K., T.K.; data curation, S.H., S.Y.Y.; writing—original draft preparation, S.H., T.K.; writing—review and editing, S.H., T.K., S.Y.H., H.Y.; visualisation, S.H.; supervision, T.K.; project administration, T.K. All authors have read and agreed to the published version of the manuscript.

Funding: No financial disclosures were reported by the authors of this paper.

Conflicts of Interest: No potential conflict of interests relevant to this article was reported. 


\section{References}

1. Abella, B.S.; Alvarado, J.P.; Myklebust, H.; Edelson, D.P.; Barry, A.; O’Hearn, N.; Hoek, T.L.V.; Becker, L.B. Quality of cardiopulmonary resuscitation during in-hospital cardiac arrest. JAMA 2005, 293, 305-310. [CrossRef]

2. Eisenberg, M.S.; Mengert, T.J. Cardiac resuscitation. N. Engl. J. Med. 2001, 344, 1304-1313. [CrossRef] [PubMed]

3. Rea, T.D.; Crouthamel, M.; Eisenberg, M.S.; Becker, L.; Lima, A.R. Temporal patterns in long-term survival after resuscitation from out-of-hospital cardiac arrest. Circulation 2003, 108, 1196-1201. [CrossRef] [PubMed]

4. Daya, M.; Schmicker, R.H.; Zive, D.M.; Rea, T.D.; Nichol, G.; Buick, J.E.; Brooks, S.; Christenson, J.; Macphee, R.; Craig, A.; et al. Out-of-hospital cardiac arrest survival improving over time: Results from the resuscitation outcomes consortium (ROC). Resuscitation 2015, 91, 108-115. [CrossRef] [PubMed]

5. Feneley, M.P.; Maier, G.W.; Kern, K.B.; Gaynor, J.W.; Gall, S.A.; Sanders, A.B.; Raessler, K.; Muhlbaier, L.H.; Rankin, J.S.; Ewy, G.A. Influence of compression rate on initial success of resuscitation and 24 hour survival after prolonged manual cardiopulmonary resuscitation in dogs. Circulation 1988, 77, 240-250. [CrossRef]

6. Meaney, P.A.; Bobrow, B.J.; Mancini, M.E.; Christenson, J.; de Caen, A.R.; Bhanji, F.; Abella, B.S.; Kleinman, M.E.; Edelson, D.P.; Berg, R.A.; et al. Cardiopulmonary resuscitation quality: [corrected] improving cardiac resuscitation outcomes both inside and outside the hospital: A consensus statement from the American Heart Association. Circulation 2013, 128, 417-435. [CrossRef]

7. Kleinman, M.E.; Brennan, E.E.; Goldberger, Z.D.; Swor, R.A.; Terry, M.; Bobrow, B.J.; Gazmuri, R.J.; Travers, A.H.; Rea, T. Part 5: Adult basic life support and cardiopulmonary resuscitation quality: 2015 American heart association guidelines update for cardiopulmonary resuscitation and emergency cardiovascular care. Circulation 2015, 132, S414-S435. [CrossRef]

8. $\quad$ Link, M.S.; Berkow, L.C.; Kudenchuk, P.J.; Halperin, H.R.; Hess, E.P.; Moitra, V.K.; Neumar, R.W.; O’Neil, B.J.; Paxton, J.H.; Silvers, S.M.; et al. Part 7: Adult advanced cardiovascular life support: 2015 american heart association guidelines update for cardiopulmonary resuscitation and emergency cardiovascular care. Circulation 2015, 132, S444-S464. [CrossRef]

9. Perkins, G.D.; Olasveengen, T.M.; Maconochie, I.; Soar, J.; Wyllie, J.; Lockey, R.G.A.; Semeraro, F.; Lott, C.; Monsieurs, K.; Nolan, J.; et al. European resuscitation council guidelines for resuscitation: 2017 update. Resuscitation 2018, 123, 43-50. [CrossRef]

10. Neumar, R.W.; Shuster, M.; Callaway, C.W.; Gent, L.M.; Atkins, D.L.; Bhanji, F.; Brooks, S.C.; de Caen, A.R.; Donnino, M.W.; Ferrer, J.M.; et al. Part 1: Executive summary: 2015 american heart association guidelines update for cardiopulmonary resuscitation and emergency cardiovascular care. Circulation 2015, 132, S315-S367. [CrossRef]

11. Muizelaar, J.P.; Marmarou, A.; Ward, J.D.; Kontos, H.A.; Choi, S.C.; Becker, D.P.; Gruemer, H.; Young, H.F. Adverse effects of prolonged hyperventilation in patients with severe head injury: A randomized clinical trial. J. Neurosurg. 1991, 75, 731-739. [CrossRef] [PubMed]

12. Sigurdsson, G.; Yannopoulos, D.; McKnite, S.H.; Lurie, K.G. Cardiorespiratory interactions and blood flow generation during cardiac arrest and other states of low blood flow. Curr. Opin. Crit. Care 2003, 9, 183-188. [CrossRef] [PubMed]

13. Aufderheide, T.P.; Sigurdsson, G.; Pirrallo, R.G.; Yannopoulos, D.; McKnite, S.; Von Briesen, C.; Sparks, C.W.; Conrad, C.J.; Provo, T.A.; Lurie, K.G.; et al. Hyperventilation-Induced Hypotension During Cardiopulmonary Resuscitation. Circulation 2004, 109, 1960-1965. [CrossRef]

14. Aufderheide, T.P.; Lurie, K.G. Death by hyperventilation: A common and life-threatening problem during cardiopulmonary resuscitation. Crit. Care Med. 2004, 32, S345-S351. [CrossRef]

15. Kleinman, M.E.; Goldberger, Z.D.; Rea, T.; Swor, R.A.; Bobrow, B.J.; Brennan, E.E.; Terry, M.; Hemphill, R.; Gazmuri, R.J.; Hazinski, M.F.; et al. 2017 american heart association focused update on adult basic life support and cardiopulmonary resuscitation quality: An update to the american heart association guidelines for cardiopulmonary resuscitation and emergency cardiovascular care. Circulation 2018, 137, e7-e13. [CrossRef]

16. Augustine, J.A.; Seidel, D.R.; McCabe, J.B. Ventilation performance using a self-inflating anesthesia bag: Effect of operator characteristics. Am. J. Emerg. Med. 1987, 5, 267-270. [CrossRef]

17. Carden, E.; Hughes, T. An evaluation of manually operated self-inflating resuscitation bags. Anesth. Analg. 1975, 54, 133-138. [CrossRef] 
18. Koga, T.; Kawamoto, M. Gender difference in mask ventilation training of anesthesia residents. J. Clin. Anesthesia 2009, 21, 178-182. [CrossRef] [PubMed]

19. McInnes, A.D.; Sutton, R.; Orioles, A.; Nishisaki, A.; Niles, D.; Abella, B.; Maltese, M.R.; Berg, R.A.; Nadkarni, V. The first quantitative report of ventilation rate during in-hospital resuscitation of older children and adolescents. Resuscitation 2011, 82, 1025-1029. [CrossRef] [PubMed]

20. O'Neill, J.F.; Deakin, C.D. Do we hyperventilate cardiac arrest patients? Resuscitation 2007, 73, 82-85. [CrossRef]

21. Kern, K.B.; Stickney, R.E.; Gallison, L.; Smith, R.E. Metronome improves compression and ventilation rates during CPR on a manikin in a randomized trial. Resuscitation 2010, 81, 206-210. [CrossRef] [PubMed]

22. Wenzel, V.; Keller, C.; Idris, A.H.; Dörges, V.; Lindner, K.H.; Brimacombe, J.R. Effects of smaller tidal volumes during basic life support ventilation in patients with respiratory arrest: Good ventilation, less risk? Resuscitation 1999, 43, 25-29. [CrossRef]

23. Kroll, M.; Das, J.; Siegler, J. Can altering grip technique and bag size optimize volume delivered with bag-valve-mask by emergency medical service providers? Prehosp. Emerg. Care 2019, 23, 210-214. [CrossRef]

24. Pirrallo, R.G.; Aufderheide, T.P.; Provo, T.A.; Lurie, K.G. Effect of an inspiratory impedance threshold device on hemodynamics during conventional manual cardiopulmonary resuscitation. Resuscitation 2005, 66, 13-20. [CrossRef] [PubMed]

25. Thayne, R.C.; Thomas, D.C.; Neville, J.D.; Van Dellen, A. Use of an impedance threshold device improves short-term outcomes following out-of-hospital cardiac arrest. Resuscitation 2005, 67, 103-108. [CrossRef] [PubMed]

26. Park, S.O. A simulation study for evaluating the efficacy of live-feedback device (Amflow) for delivering correct ventilations during cardiopulmonary resuscitation. J. Korean Soc. Emerg. Med. 2017, 28, 159-166.

27. You, K.M.; Lee, C.; Kwon, W.Y.; Lee, J.C.; Suh, G.J.; Kim, K.S.; Park, M.J.; Kim, S. Real-time tidal volume feedback guides optimal ventilation during simulated CPR. Am. J. Emerg. Med. 2016, 35, 292-298. [CrossRef]

28. Ambu®Mark IV-Reusable resuscitator datasheet. Available online: https://www.ambu.com/products/ anaesthesia/resuscitators/product/ambu-mark-iv.;2019 (accessed on 10 January 2020).

29. Elling, R.; Politis, J. An evaluation of emergency medical technicians' ability to use manual ventilation devices. Ann. Emerg. Med. 1983, 12, 765-768. [CrossRef]

30. Lee, M.J.; Rho, T.-H.; Kim, H.; Kang, G.H.; Kim, J.S.; Rho, S.G.; Park, H.K.; Oh, D.J.; Oh, S.; Wi, J.; et al. Part 3. Advanced cardiac life support: 2015 Korean Guidelines for Cardiopulmonary Resuscitation. Clin. Exp. Emerg. Med. 2016, 3, S17-S26. [CrossRef]

31. Khoury, A.; De Luca, A.; Sall, F.; Pazart, L.; Capellier, G. Performance of manual ventilation: How to define its efficiency in bench studies? A review of the literature. Anaesthesia 2015, 70, 985-992. [CrossRef]

32. Terndrup, T.; Rhee, J. Available ventilation monitoring methods during pre-hospital cardiopulmonary resuscitation. Resuscitation 2006, 71, 10-18. [CrossRef] [PubMed]

33. Siegler, J.; Kroll, M.; Wojcik, S.; Moy, H.P. Can EMS providers provide appropriate tidal volumes in a simulated adult-sized patient with a pediatric-sized bag-valve-mask? Prehosp. Emerg. Care 2017, 21, 74-78. [CrossRef] [PubMed]

34. Doerges, V.; Sauer, C.; Ocker, H.; Wenzel, V.; Schmucker, P. Smaller tidal volumes during cardiopulmonary resuscitation: Comparison of adult and paediatric self-inflatable bags with three different ventilatory devices. Resuscitation 1999, 43, 31-37. [CrossRef]

(C) 2020 by the authors. Licensee MDPI, Basel, Switzerland. This article is an open access article distributed under the terms and conditions of the Creative Commons Attribution (CC BY) license (http://creativecommons.org/licenses/by/4.0/). 